\title{
Impacto da atividade física sobre a incontinência urinária - Revisão sistemática
}

\author{
Impact of physical activity on urinary incontinence - Systematic review
}

Impacto de la actividad física en la incontinencia urinaria - Revisión sistemática

\author{
Glaucineide Araújo Nunes de Souza ${ }^{\mathrm{I}}$, Fabiana Coalho Lino Marchesi ${ }^{\mathrm{II}}$, Loraine Laisa Gonçalves \\ Mazeto ${ }^{\mathrm{III}}$, Erica Feio Carneiro Nunes ${ }^{\mathrm{IV}}$, Gustavo Fernando Sutter Latorre ${ }^{\mathrm{V}}$
}

\begin{abstract}
Resumo
O objetivo deste estudo foi discutir o impacto da atividade física sobre a incontinência urinária feminina. Foi realizada uma revisão sistemática de ensaios clínicos, usando as bases de dados PUBMED, LILACS, PEDro e Scielo. Utilizou-se os descritores atividade física, esporte, incontinência urinária e mulheres para a busca. Foram incluídos estudos publicados entre 2005 e 2017, sendo excluídos ensaios em cobaias e duplicados nas bases. Os resultados mostraram que atividades de alto impacto estão relacionadas à incontinência urinária. A maior prevalência foi em praticantes de Jump de 25 a $76 \%$. A prevalência geral de incontinência urinária em praticantes de atividade física ou desporto oscilou de 12,5 a $76 \%$. Desta forma, conclui-se que a prática de atividade física impacta negativamente sobre o assoalho pélvico, e atividades de alto impacto são fortemente correlacionadas à incontinência urinária.
\end{abstract}

Palavras-chaves: Incontinência Urinária; Mulheres; Exercício

\begin{abstract}
The objective of this study was to discuss the impact of physical activity on female urinary incontinence. A systematic review of clinical trials was performed using the PUBMED databases, LILACS, PEDro and Scielo. Physical activity descriptors, sport, urinary incontinence and women for the search were used. Studies published between 2005 and 2017 were included, being excluded trials in guinea pigs and duplicates on the bases. The results showed that high impact activities are related to urinary incontinence. The highest prevalence was in Jump practitioners from 25 to $76 \%$. The general prevalence of urinary incontinence in practitioners of physical activity or sport oscillated from 12.5 to $76 \%$. In this way, it is concluded that the practice of physical activity negatively impacts on the pelvic floor, and high impact activities are strongly correlated with urinary incontinence.
\end{abstract}

Keywords: Urinary incontinence; Women; Exercise

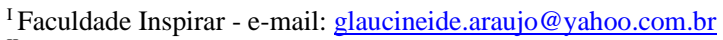

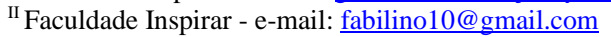

${ }^{\text {III }}$ Faculdade Inspirar - e-mail: lolaisagoncalves@ gmail.com

IV Universidade do Estado do Pará- e-mail: erica@perineo.net

${ }^{\vee}$ Faculdade Inspirar - Rua Silva Jardim, 307 - Centro - Florianópolis, SC, Brasil - e-mail: gustavo@perineo.net
} 


\section{Resumen}

El objetivo de este estudio fue discutir el impacto de la actividad física en la incontinencia urinaria femenina. Se realizó una revisión sistemática de los ensayos clínicos utilizando las bases de datos PUBMED, LILACS, PEDro y Scielo. Para la búsqueda se utilizaron los descriptores actividad física, deporte, incontinencia urinaria y mujeres. Se incluyeron estudios publicados entre 2005 y 2017, siendo excluidos los ensayos en cobayas y duplicados en las bases. Los resultados mostraron que las actividades de alto impacto están relacionadas con la incontinencia urinaria. La prevalencia más alta fue en practicantes de jump del 25 al $76 \%$. La prevalencia general de la incontinencia urinaria en los practicantes de actividad física o deporte osciló de 12.5 a $76 \%$. De esta manera, se concluye que la práctica de la actividad física afecta negativamente en el suelo pélvico, y las actividades de alto impacto están fuertemente correlacionadas con la incontinencia urinaria.

Palabras clave: Incontinencia urinaria; Mujeres; Ejercicio

\section{Introdução}

A incontinência urinária (IU) é definida pela Sociedade Internacional de Continência como qualquer perda involuntária de urina. Os fatores de risco para IU são: idade avançada, obesidade, gestação, parto vaginal com lesão de nervos periféricos, de fáscias e de ligamentos, deficiência estrogênica (menopausa), tabagismo, doenças do colágeno, fragilidade do tecido conjuntivo e dos músculos do assoalho pélvico, neuropatias e histerectomia prévia (BARROS; LUCENA; ANSELMO, 2007).

Na última década, a IU tem sido um problema muito comum entre mulheres nulíparas, jovens e praticantes de atividade física (MARTINS et al., 2017), pois exercícios físicos que exigem bastante esforço físico e demandam alto impacto podem acarretar em incontinência urinária de esforço (IUE) (CAETANO; TAVARES; LOPES, 2007). Dessa forma, o exercício físico se transforma em um fator de risco para a IUE em mulheres atletas, principalmente aquelas que não têm histórico de partos e gestações.

A IU durante a atividade esportiva em atletas de elite apresenta uma incidência que varia de $0 \%$ (golfe) a 80\% (trampolim) (ALMEIDA et al., 2011). Ocorre com maior frequência entre jovens que realizam alguma atividade que envolvem longos saltos, pois apresentam alto impacto e causam maior pressão sobre a musculatura de assoalho pélvico (MAP) (ARAUJO et al.,2015).

Algumas teorias tentam esclarecer a etiologia da IUE em mulheres atletas: 1) apesar das atletas apresentarem MAP fortalecida, o exercício físico intenso poderia causar a elevação da pressão abdominal, predispondo a IUE; 2) as atletas apresentam sobrecarga, estiramento e enfraquecimento da MAP; 3) a amenorreia hipotalâmica, decorrente da intensa atividade física, distúrbios alimentares ou a associação de ambos, devido à diminuição dos níveis de estrogênio, também poderia favorecer o surgimento da IUE 
nessas mulheres (ARAÚJO et al., 2008); 4) atividades de alto impacto podem afetar o processo de continência devido ao aumento da quantidade de força transmitida para a MAP (PATRIZZI et al., 2014).

Considerando a importância de se trabalhar a musculatura do assoalho pélvico em mulheres fisicamente ativas para evitar o surgimento de IU, esta revisão tem como objetivo discutir o impacto da atividade física sobre a incontinência urinária feminina, por meio de uma revisão sistemática.

\section{Metodologia}

Trata-se de uma revisão sistemática, construída a partir do levantamento em bancos de dados eletrônicos da Scientific Electronic Library Online (SciELO), National Library of Medicine and National Institutes of Health (PubMed) Literatura Latino-Americana e do Caribe em Ciências da Saúde (LILACS) e Physiotherapy Evidence Database (PEDro). Utilizaram-se os descritores incontinência urinária, mulheres e exercícios físicos, bem como suas variantes em inglês e respectivas associações.

Foram incluídos estudos publicados no intervalo compreendido entre 2005 a 2017, que, de alguma forma, cruzaram dados sobre incontinência urinária e alguma atividade física em mulheres. Foram excluídos estudos em animais e em homens.

O processo de seleção foi conduzido por duas pesquisadoras independentes, e as divergências resolvidas em reunião de consenso. Os estudos encontrados foram selecionados pelo título, em seguida, pelo resumo e, por fim, pelo texto na íntegra, e classificados em uma tabela por autor, ano, tipo de incontinência urinária e tipo de atividade física. O escore PEDro de cada estudo foi apresentado entre colchetes e após a identificação do autor principal e ano, na próxima sessão, de resultados.

As buscas eletrônicas nas bases de dados Pubmed, LILACS, PEDro e Scielo, perfizeram um total bruto de 3564 artigos, com 2030 artigos na base Pubmed, 882 na LILACS, 610 na PEDro e 42 na base Scielo. Entretanto, após aplicados os critérios de elegibilidade do estudo, foram selecionados 8 artigos para compor a presente revisão

\section{Resultados}

Foram selecionados 8 artigos para compor a presente revisão (Figura 1). Os artigos são apresentados com a nota obtida na escala PEDro entre colchetes. 
Figura 1: Fluxograma com o processo de seleção, inclusão e exclusão dos artigos primários.

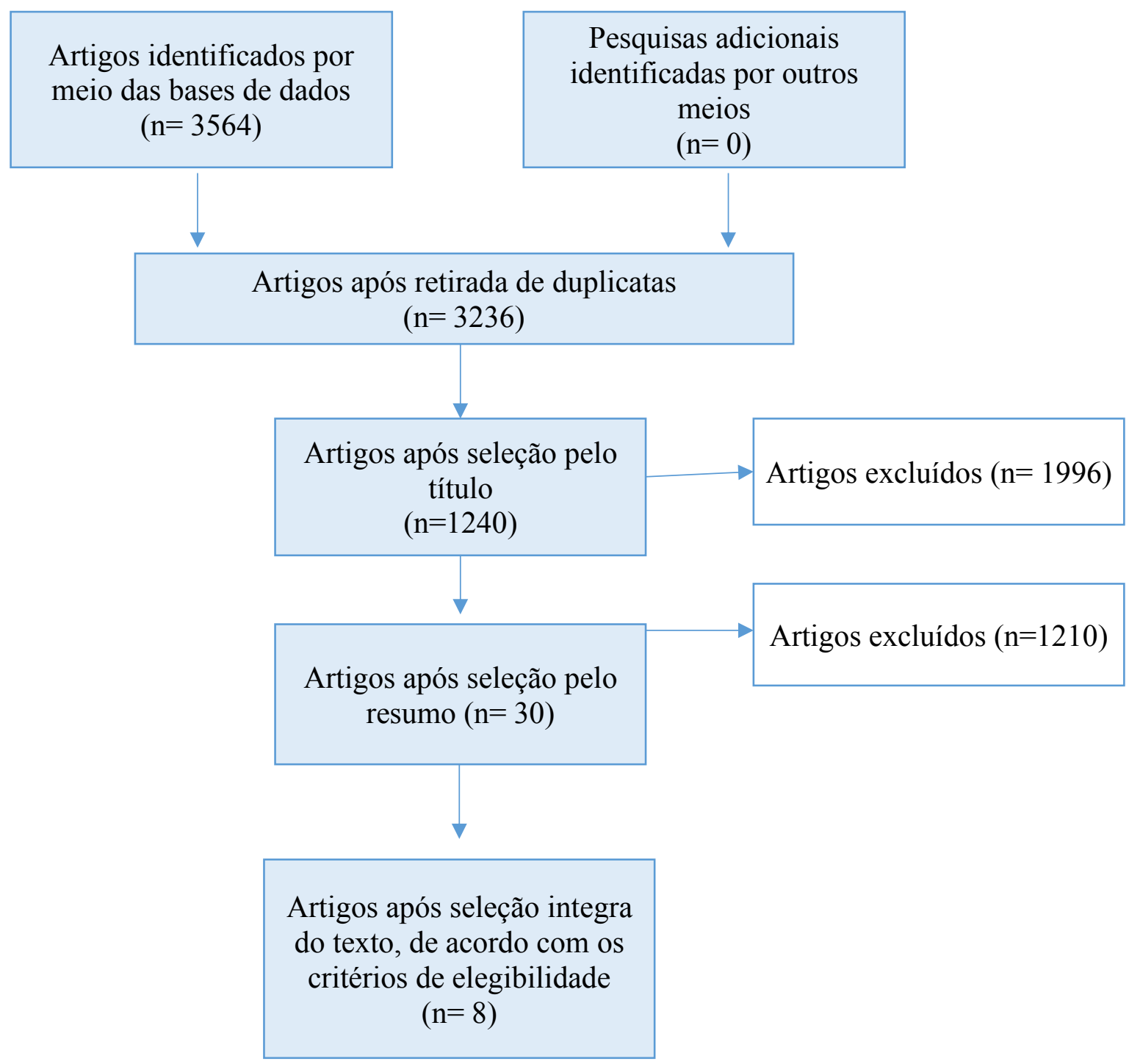

Fonte: dos autores.

Araújo et al. (2008) [3/10], estudaram a função urinária de 37 corredoras de longa distância, que perfaziam treinos diários de pelo menos uma hora e que participavam de competições regulares. As corredoras realizaram pad test e completaram os questionários International Consultation on Incontinence Questionnaire - Short Form (ICIQ-SF), que avalia os sintomas de IU, e o Eating Attitudes Test (EAT-26), sobre hábitos alimentares. Ao final do estudo, constatou-se que 23 atletas $(62,2 \%)$ apresentaram queixas de perda de urina, sendo que cerca de 60,9\% delas apresentavam IU durante o treino e $65,2 \%$ perdiam urina durante a competição. 
Almeida e Machado (2012) [3/10] estudaram 32 voluntárias de um grupo de mulheres praticantes de jump. As mesmas responderam ao questionário sobre histórico obstétrico e rotinas da atividade física e ao questionário ICIQ-SF. Houve relatos de perda urinária por 37,5\% das entrevistadas, em que as praticantes da atividade três ou mais vezes na semana apresentaram 2,45 mais chances de apresentar IU.

Fozzatti et al. (2012) [6/10] avaliaram 488 atletas divididas em dois grupos. Para a avaliação, foram utilizados ICIQ-SF, e um questionário específico sobre a atividade física, com tipo e frequência de exercício e a relação entre a perda de urina e a atividade. O primeiro grupo com atletas de diversas modalidades esportivas, dentre elas caminhada, corrida, ginástica abdominal, jump, ciclismo, exercícios localizados, step, alongamento, hidroginástica, Pilates, musculação e natação; o segundo grupo com mulheres que não frequentavam academias. Ao final, 24,6\% das mulheres que praticavam atividades físicas avaliadas relataram IU. O jump foi a atividade que mais levou a perda de urina (25\%).

Patrizzi et. al. (2014) [5/10] dividiram 108 atletas de faixa etária de 18 a 30 anos, nulíparas, praticantes de exercícios físicos com frequência de três dias ou mais por semana e duração de no mínimo uma hora, em três grupos: G1 de musculação, G2 de exercício aeróbico e G3 de natação, avaliando a função urinária por um questionário desenvolvido pelas autoras. A perda urinária esteve presente nas mulheres questionadas durante a prática de exercício físico com percentual de 42,5\%. Evidenciaram-se maiores queixas de IU no G1, quando comparado com o G3 (p=0,01). Os resultados sugerem maior proporção de IU em praticantes de musculação, quando comparadas a praticantes de exercícios aeróbicos $(\mathrm{p}=0,09)$ e, não houve diferença estatística $(\mathrm{p}=0,12)$ entre as praticantes de exercícios aeróbicos, quando comparadas às praticantes de natação.

Roza et al. (2015) [6/10] investigaram 386 estudantes com idade entre 14 e 33 anos, divididas em quatro grupos, G1: mulheres sedentárias ou que praticavam de 0 a 30 minutos por semana, consideradas fisicamente inativas; G2: mulheres praticantes de atividade física moderada com variação de 31 a 180 minutos de exercícios por semana; G3: estudantes de educação física que praticavam de 181 a 420 minutos de exercícios por semana; e G4: atletas profissionais que praticavam de 421 a 940 minutos por semana, tendo como base as recomendações dos critérios do Colégio Americano de Medicina Esportiva (ACSM). Todas elas realizavam diferentes disciplinas esportivas, como o trampolim, futebol, handebol, natação, voleibol, atletismo e artes marciais. Para avaliar os sintomas de IU, os autores utilizaram um questionário composto por 13 itens para análise das características sociodemográficas, história médica, obstétrica, e o questionário ICIQ-SF. Ao final do estudo, 19,9\% das participantes relataram perda de urina, para todos os grupos. A perda urinária foi mais frequente nas atletas profissionais, que apresentaram um risco relativo $2,53 \%$ maior, quando comparadas com mulheres que não praticavam 
atividade física. Ao comparar as atletas com as mulheres que praticavam atividade moderada, o risco foi $0,8 \%$ maior para as atletas, que também apresentaram risco $1 \%$ maior na prevalência de IU quando comparadas às estudantes de educação física.

Roza et al. (2015) [3/10], analisaram 22 atletas do sexo feminino durante o campeonato nacional Português de trampolim. Antes da competição, foi realizado um questionário com duas sessões de questões, uma relacionada com a vida esportiva das voluntárias, dados antropométricos, menarca, escolaridade, fase da vida em que apareceram os sintomas de IUE e rotina de treino no trampolim; e outra sessão com o ICIQ-SF. Ao término da competição foi estabelecida a relação entre perda urinária e a classificação das atletas de elite, levando em consideração que as esportistas com melhor pontuação são as que passam por treinos mais desgastantes. A atividade física foi dividida em tercis de volume de treino (horas de treino por semana $\mathrm{x}$ anos de prática). Ao final, cerca de 72,7\% das participantes informaram ter perda de urina durante a prática de jump sendo descrito que os episódios de perda começaram após o início desse tipo de prática desportiva.

Araújo et al. (2015) [6/10] estudaram a função urinária de 93 mulheres, dentre as 49 atletas e 44 sedentárias. As atletas eram praticantes de basquete, ginástica olímpica e corrida de longa distância. As sedentárias realizavam no máximo 150 minutos por semana de atividades moderadas ou vigorosas. Para as medidas, os autores utilizaram o ICIQ-SF. Os resultados evidenciaram a prevalência de incontinência urinária nas atletas de $76 \%$, contra $16 \%$ nas sedentárias $(\mathrm{p}=0,005)$.

Finalmente, Rosa et al. (2016) [4/10] estudaram 59 mulheres, praticantes de jump por um período de cerca de cinco meses e com frequência semanal de três vezes na semana. Para as medidas, utilizaram o King's Health Questionnaire, com o objetivo de avaliar o impacto da IU na qualidade de vida. Houve uma prevalência de 66,1\% de incontinência urinaria nas mulheres estudadas. Quanto à qualidade de vida, os autores descreveram que, quanto maior o período da atividade física, menor a percepção total de saúde $(\mathrm{r}=0,340 ; \mathrm{p}<0,05)$. Ainda, a prática do jump aumentou as restrições funcionais em atividades de vida diária $(\mathrm{r}=0,368 ; \mathrm{p}<0,05)$ e a restrição física $(\mathrm{r}=0,328 ; \mathrm{p}<0,05)$. Também, os autores verificaram que atletas praticantes da modalidade por mais de 3 meses aumentaram o risco de IU em 13,38 vezes, corroborando as observações de que, quanto maior período de pratica física, menor a qualidade de vida em mulheres com perdas urinárias aos esforços. 


\section{Discussão}

O presente estudo objetivou discutir o impacto da atividade física sobre a IU feminina. De modo geral, a prática desportiva esteve associada à IU nos estudos. As modalidades descritas foram natação, musculação, caminhada, corrida, ginástica abdominal, jump, ciclismo, exercícios localizados, step, alongamento, hidroginástica, pilates, futebol, handebol, natação, voleibol, atletismo, artes marciais, basquete, ginástica olímpica e corrida de longa distância. Dentre elas, a modalidade que apresentou maior prevalência de IU foi o jump com prevalência oscilando entre 25 e $76 \%$.

Fozzatti et al (2012) estudaram a ocorrência de IU em 488 mulheres divididas entre as que praticavam academia e as que praticavam esporte, observando que a frequência de IU no jump foi de $25 \%$, seguida de step $23,3 \%$, corrida $19,5 \%$, abdominal $16,1 \%$, exercício localizado $15,1 \%$, hidroginástica $14,3 \%$, musculação $14 \%$, alongamento $13,2 \%$, caminhada $12,1 \%$, bicicleta $10,1 \%$, natação $7,1 \%$ e pilates $5,6 \%$. Ressalta-se que a maioria de mulheres eram jovens (média de 25,68 anos no grupo de esporte e 22,8 anos no grupo academia).

Quanto ao tipo de IU, o mais comumente descrito em desportistas é a IU por esforço, seguida de IU mista e IU de urgência (Roza et al., 2015). As modalidades de esporte que incluem salto, como o jump, são as atividades físicas que impactaram negativamente a função urinária. Essa observação poderia ser explicada pelo fato de que as contrações dos músculos do assoalho pélvico, por repetição, causadas pelo impacto, afetariam negativamente a função do assoalho pélvico, cuja consequente falência desencadearia disfunções sobre as três funções fisiológicas: evacuatória, sexual e urinária. De fato, atividades classificadas como alto impacto apresentam maiores chances de escapes urinários, dentre estas o jump, o atletismo e a ginástica, uma vez que as forças de reação do solo no decorrer do pouso vertical máximo podem atingir 16 vezes a carga corporal da praticante. Por esse motivo, modalidades envolvendo o salto são correlacionadas a maiores prevalências de IU (MARTINS et al., 2017). Em diferentes atividades esportivas, a força de impacto corresponde de três a quatro vezes o peso corporal durante a corrida; em pulos, cerca de 5 a 12 vezes; e de 9 vezes durante a realização de salto com vara (ALMEIDA et al., 2011).

Atualmente, devido à prática de atividade física estar se tornando mais frequente na vida das mulheres, as elevações bruscas da pressão intra-abdominal relativas à essas modalidades desportivas pode ser um fator de ameaça para o surgimento de IU de esforço (REIS et al., 2011). Por isso, estratégias preventivas devem ser traçadas e implementadas, visando esses grupos de risco específicos, formados por mulheres que praticam atividades de impacto. 
O exercício físico promove melhoria na saúde em geral e na qualidade de vida; por outro lado, o esporte, que visa não à saúde, mas ao resultado, focado, portanto, no rendimento, pode gerar consequências para a saúde, tanto pelo excesso de carga, quanto pelos mecanismos de repetição gestual (ROZA, 2011).

São descritas diversas lesões associadas ao desporto de rendimento. Particularmente, as disfunções do assoalho pélvico têm sido relatadas recentemente em mulheres atletas. Embora essa disfunção ocorra com mais frequência em mulheres com idade mais avançada, alguns estudos mais recentes têm citado a perda involuntária de urina em atletas nulíparas e jovens. Isso acontece com maior prevalência em esportes que envolvem atividades de alto impacto, por exemplo a ginástica, o atletismo e alguns jogos desportivos (ROZA, 2011). Os dados da presente revisão corroboram esta inquietude presente na literatura, tornando urgente, mais uma vez, a implementação de estratégias preventivas específicas.

A IU causa restrições na vida social das mulheres acometidas, como o abandono ou a diminuição das atividades físicas, e mesmo atividades em geral, como frequentar lugares públicos, viajar e visitar amigos, dentre várias outras atividades de lazer. Isso acontece pelo constrangimento que a IU causa, além do medo relacionado ao estar "suja de urina".

Como limitação, aponta-se a carência de estudos epidemiológicos sobre o tema, uma vez que esta revisão teve de ser construída sobre os oito estudos hoje disponíveis, mesmo lançando mão de critérios de seleção amplos.

Deste modo, o presente conhecimento e estado da arte abrem importante horizonte de prevenção para mulheres que praticam atividades desportivas, especialmente aqueles exercícios relacionados ao impacto, além de fornecer as melhores ferramentas da atualidade para o tratamento de possíveis sequelas já instaladas, por conta da falência do assoalho pélvico, relacionada à prática desportiva.

\section{Conclusão}

Modalidades desportivas de alto impacto, em especial atividades com salto, estão relacionadas com a queixa de perda de urina. A IU gera sentimentos de medo, vergonha e constrangimento, consequentemente tendendo ao isolamento social as pessoas que sofrem desse acometimento. Nas praticantes de atividade física, soma-se ainda a queda no rendimento ou até mesmo o abandono da prática desportiva. 
Apesar da relevância social do tema, são escassos os estudos longitudinais a respeito das disfunções do assoalho pélvico em mulheres atletas, e, considerando a amplitude dos impactos sobre a saúde e qualidade de vida dessa população, bem como a qualidade metodológica de média para baixa dos estudos presentes, novos estudos nesse sentido se fazem urgentes.

\section{Referências}

ALMEIDA, M. B. A. et al., Disfunções de assoalho pélvico em atletas. Revista FEMINA, vol.39, n.8, pág. 395-402, 2011.

ALMEIDA, P. P.; MACHADO, L. R. G., A prevalência de incontinência urinária em mulheres praticantes de jump. Fisioterapia e Movimento, vol.25, n.1, pág.55-65, 2012.

ARAUJO, M.P. et al., Avaliação do Assoalho Pélvico De Atletas: existe Relação Com A Incontinência Urinária? Revista Brasileira de Medicina do Esporte, vol.21, n.6, 2015.

ARAÚJO, M. P. et al., Relação entre incontinência urinária em mulheres atletas corredoras de longa distância e distúrbio alimentar. Revista da Associação Médica Brasileira, vol.54, n.2, pág. 146-149, 2008.

BARROS, J. D.; LUCENA, A. C. T.; ANSELMO, C. W. S. F., Incontinência urinária de esforço em atletas do sexo feminino: uma revisão da literatura. Anais da Faculdade de Medicina da Universidade Federal de Pernambuco, vol. 52, n.2, pág. 173-180, 2007.

BO, K. et al. Evidence-Based Physical Therapy for the Pelvic Floor: Bridging Science and Clinical Practice. 2 ed. London: Churchill Livingstone, 2015, 446 p.

CAETANO, A. S.; TAVARES, M. C. G. C. F.; LOPES, M. H. B. M., Revista Brasileira de Medicina e Esporte, vol.13, n.4, pág. 270-274, 2007.

FOZZATTI C, et. al. Prevalence study of stress urinary incontinence in women who perform high-impact exercises. Int Urogynecol J. v. 23, pág.1687-1691, 2012.

LEITE, J. C. et al., Avaliação funcional do assoalho pélvico: uma abordagem fisioterapêutica na prevenção de incontinência urinária. Fisioterapia Brasil, vol.13, n.6, pág.67-72, 2012.

MARTINS, L.A. et al., A perda de urina é influenciada pela modalidade Esportiva ou pela carga de treino? Uma revisão sistemática. Revisa Brasileia de Medicina do Esporte. vol. 23, n.1, 2017.

PATRIZZI, L. J. et al., Incontinência urinária em mulheres jovens praticantes de exercício físico. Revista Brasileira Ciência e Movimento, vol.22, n.3, pág.105-110, 2014.

REIS, A. O. et al., Estudo comparativo da capacidade de contração do assoalho pélvico em atletas de voleibol e basquetebol. Revista Brasileira de Medicina e Esporte, vol.17, n.2, pág.97-101, 2011. 
RETT, M. T. et al., Qualidade de vida em mulheres após tratamento de incontinência urinária de esforço com fisioterapia. Revista Brasileira de Ginecologia e Obstetrícia, vol.29, n.3, pág.134-140, 2007.

ROZA, T. H. Prevalência de incontinência urinária feminina e proposta de um protocolo de reabilitação funcional dos músculos do pavimento pélvico para mulheres atletas. 2011. 150 págs. Faculdade de Desporto da Universidade do Porto.

ROSA, P.V. et al. Prevalência de incontinência urinária em mulheres praticantes de jump. Fisioterapia Brasil. vol.17, n.1, 2016.

ROZA, T. et. Al. Volume of Training and the Ranking Level Are Associated With the Leakage of Urine in Young Female Trampolinists. Clin J Sport Med. vol. 25, n.3, 2015.

ROZA,T. et al., Urinary Incontinence and Levels of Regular Physical Exercise in Young Women. Int J Sports Med. v. 36, p. 776-780, 2015.

SILVA, D. T. G.; MORAES, N. M., Estudo comparativo da força muscular do assoalho pélvico em mulheres sedentárias e mulheres que praticam atividade física. Universidade da Amazônia, Belém, Pará, 2006.

SILVA, L. H. et al., Relação da incontinência urinária de esforço com a prática de atividade física em mulheres nulíparas. Revista Salusvita, vol.24, n.2, pág. 195-206, 2005.

\section{Como citar este artigo}

SOUZA, G. A. N.; MARCHESI, F. C. L.; MAZETO, L. L. G.; NUNES, E. F. C.; LATORRE, G. F. S. Impacto da atividade física sobre a incontinência urinária - Revisão sistemática. Revista Kinesis, Santa Maria, v. 39, p.01-10, 2021.

* O presente trabalho não contou com apoio financeiro de nenhuma natureza para sua realização. 